\title{
Histone methyltransferase KMT5A gene modulates oncogenesis and lipid metabolism of papillary thyroid cancer in vitro
}

\author{
TIAN LIAO ${ }^{1 *}$, YUAN-JIN WANG ${ }^{2 *}$, JIA-QIAN HU $^{1 *}$, YU WANG $^{1}$, LI-TAO HAN $^{1}$, BEN MA $^{1}$, \\ RONG-LIANG SHI ${ }^{1}$, NING QU ${ }^{1}$, WEN-JUN WEI ${ }^{1}$, QING GUAN ${ }^{1}$, JUN XIANG ${ }^{1}$, \\ JIA-YING CHEN ${ }^{1}$, GUO-HUA SUN ${ }^{1}$, DUAN-SHU LI ${ }^{1}$, XIANG-MING MU ${ }^{2}$ and QING-HAI JI ${ }^{1}$ \\ ${ }^{1}$ Department of Head and Neck Surgery, Shanghai Cancer Center, Department of Oncology, Shanghai Medical College, \\ Fudan University, Shanghai 200032; ${ }^{2}$ Department of General Surgery, Yancheng First People's Hospital, \\ The Fourth Affiliated Hospital of Nantong Medical College, Yancheng, Jiangsu 224000, P.R. China
}

Received January 21, 2017; Accepted September 8, 2017

DOI: $10.3892 /$ or.2018.6295

\begin{abstract}
KMT5A (known as PR-Set7/9, SETD8 and SET8), a member of the SET domain containing methyltransferase family specifically targeting H4K20 for methylation, has been implicated in multiple biological processes. In the present study, we identified that KMT5A was elevated in 50 pairs of papillary thyroid cancer tissue samples and in cell lines K1 and TPC-1 by qRT-PCR and western blotting, as well as by immunohistochemical staining. CCK-8 assay and flow cytometric analysis revealed that inhibition of KMT5A attenuated proliferation and induced apoptosis. Transwell assays revealed that cell migration and invasion were suppressed in KMT5Aknockdown cells. Moreover, the inhibition of KMT5A arrested the cell cycle in the G1/S phase of papillary thyroid cancer cells. The TCGA data revealed that elevated KMT5A expression was significantly correlated with extrathyroidal extension, lymph node metastasis and advanced pathological stage of papillary thyroid cancer. Furthermore, we observed that inhibition of KMT5A suppressed the expression of SREBP1, SCD, FASN and ACC, key molecules involved in lipid metabolism and decreased the level of malondialdehyde in papillary thyroid cancer cells. In conclusion, KMT5A may be a novel oncogenic factor, specifically a regulator for lipid metabolism in papillary thyroid carcinoma.
\end{abstract}

\section{Introduction}

Thyroid cancer is the most common endocrine tumor and the incidence of thyroid cancer is rising by $4 \%$ every year,

Correspondence to: Professor Qing-Hai Ji, Department of Head and Neck Surgery, Shanghai Cancer Center, Department of Oncology, Shanghai Medical College, Fudan University, Shanghai 200032, P.R. China

E-mail: ji_qh2015@163.com

${ }^{*}$ Co-first authorship

Key words: KMT5A, papillary thyroid cancer, lipid metabolism approximately $80 \%$ of which accounts for papillary thyroid carcinoma (PTC) (1). Although PTC is a highly curable disease and its mortality rate is low, nearly $10-20 \%$ of PTCs are progressive with aggressive tumor behavior and high disease recurrence (2). The underlying factors and mechanisms for the aggressiveness of PTC remain unclear.

Recently, research has shed light on the importance of fatty acid metabolism in cancer progression (3). The alteration of fatty acid metabolism influences the energy stores, affects drug resistance, modulates cell proliferation and survival and stimulates the extracellular environment $(4,5)$. Some studies have revealed that transformed cells and malignant tumors exhibit overexpression of elements involved in de novo lipid metabolism $(6,7)$. This increased lipid biosynthesis in tumor tissue may offer a new insight into the molecular mechanisms that manage the progression of malignant tumors.

KMT5A, known as SET8, PR-Set7/9 and SETD8, a member of the SET domain containing methyltransferase family specifically targeting H4K20 for monomethylation, has been reported to play key roles in diverse biological processes, including gene transcriptional control, replication origin modulation, genome integrity maintenance and cell cycle progression and development (8-13). Accumulating evidence revealed that SET8 may participate in tumor development and progression $(14,15)$. However, whether KMT5A is involved in the oncogenesis of PTC is currently not known.

In the present study, we found that KMT5A was overexpressed in PTC tissues as well as in PTC cells. Knockdown functional analysis revealed that KMT5A participated in the proliferation, apoptosis, cell cycle, migration and invasion of PTC cells. Upon further research we found that downregulation of KMT5A also attenuated fatty acid metabolism in in vitro models of PTC. These results provide mounting evidence of KMT5A as an oncogenic mediator in fatty acid metabolism of PTC.

\section{Materials and methods}

Tissue specimens. Fifty pairs of papillary thyroid cancer samples consisting of 50 PTCs and 50 matched normal thyroid tissues ( $1 \mathrm{~cm}$ away from the edge of the tumor tissue) were 
obtained between 2012 and 2015 from patients who underwent thyroid cancer surgery at the Shanghai Cancer Center in Fudan University. Tissue specimens were frozen in liquid nitrogen immediately after surgical resection and stored at $-80^{\circ} \mathrm{C}$. Final histological classification was based on paraffin-embedded sections. The use of clinical materials for research purposes in the present study was approved by the Institutional Research Ethics Committee of Fudan University prior to the obtained written consent of patients.

The KMT5A gene expression and clinical data from 455 papillary thyroid carcinomas in the TCGA database are available from the website www.cbioportal.org/. The relationship between KMT5A and the clinical parameters was analyzed using the TCGA data (Table I).

Cell lines and transfections. Human thyroid follicular epithelial cell line Nthy-ori 3-1 and human papillary thyroid cancer cell lines K1 and TPC-1 were grown in RPMI-1640 media (Gibco, Carlsbad, CA, USA) supplemented with $10 \%$ fetal bovine serum (FBS; Invitrogen, Carlsbad, CA, USA) and $1 \%$ penicillin-streptomycin $(10,000 \mathrm{U} / \mathrm{ml}$ penicillin and $10 \mathrm{mg} / \mathrm{ml}$ streptomycin in $0.9 \% \mathrm{NaCl}$; Sigma-Aldrich, St. Louis, MO, USA) at $37^{\circ} \mathrm{C}$ with $5 \% \mathrm{CO}_{2}$. We constructed a lentiviral system to knock down KMT5A: HEK-293T cells were co-transfected with pLKO.1-KMT5A 1 and 2 (Biotend, Shanghai, China) using Lipofectamine 2000 (Invitrogen, Shanghai, China). Forty-eight hours after transfection, the virus-containing medium collected from HEK-293T cells was used to infect PTC cells K1 and TPC-1 to establish KMT5A-shRNA cells, followed by $2 \mu \mathrm{g} / \mathrm{ml}$ puromycin selection (Sigma-Aldrich). Human thyroid follicular epithelial cell line Nthy-ori 3-1 was obtained from Sigma-Aldrich. Human papillary thyroid cancer cell lines K1 and TPC-1 were purchased from the University of Colorado Cancer Center Cell Bank.

RNA extraction and RT-PCR. Total RNA of tissue or cell lines was extracted using TRIzol reagent (Invitrogen). Total RNA $(1 \mu \mathrm{g})$ was used for cDNA synthesis employing PrimeScript ${ }^{\mathrm{TM}}$ RT Reagent kit (Takara Bio, Inc., Shiga, Japan). RT-PCR was performed in triplicate with SYBR Premix Ex Taq ${ }^{\mathrm{TM}}$ kit (Takara Bio) according to the manufacturer's instructions. The primers for the target genes were synthesized by Sangon Biotech Co., Ltd. (Shanghai, China) (Table II). The thermal cycling parameters were: $95^{\circ} \mathrm{C}$ for $30 \mathrm{sec}, 95^{\circ} \mathrm{C}$ for $5 \mathrm{sec}$ and $60^{\circ} \mathrm{C}$ for $30 \mathrm{sec}$. $\beta$-actin was used as an internal control. The threshold cycle $(\mathrm{Ct})$ values were analyzed using the comparative $\mathrm{Ct}\left(2^{-\Delta \Delta \mathrm{Ct}}\right)$ method. The level of target genes was calculated by normalizing to the endogenous reference and relative to a control (16).

Immunohistochemicalstaining. Immunohistochemical staining (IHC) was carried out according to the manufacturer's protocol. Briefly, formalin-fixed and paraffin-embedded tissue sections were deparaffinized in xylene and hydrated through descending concentrations of ethanol before being placed in a blocking solution to inhibit endogenous peroxidase activity. The slides were incubated with primary antibodies (rabbit anti-human KMT5A; dilution, 1:200; Cell Signaling Technology, Shanghai, China ) at $4^{\circ} \mathrm{C}$ overnight. HRP-conjugated rabbit secondary antibody (dilution, 1:100; Cell Signaling Technology) was
Table I. Relationship between the KMT5A expression and the clinicopathological parameters of the PTC patients.

\begin{tabular}{|c|c|c|c|}
\hline \multirow{2}{*}{$\begin{array}{l}\text { Clinicopathological } \\
\text { parameters }\end{array}$} & \multicolumn{2}{|c|}{ KMT5A expression } & \multirow[b]{2}{*}{ P-value } \\
\hline & Low, n (\%) & High, $n(\%)$ & \\
\hline Age (years) & & & 0.769 \\
\hline$<45$ & $77(36.5)$ & $134(63.5)$ & \\
\hline$\geq 45$ & $85(35.0)$ & $158(65.0)$ & \\
\hline Sex & & & 0.259 \\
\hline Male & $38(40.9)$ & $55(59.1)$ & \\
\hline Female & $92(34.5)$ & $179(65.5)$ & \\
\hline Tumor size $(\mathrm{cm})$ & & & 0.29625 \\
\hline$\leq 1$ & $23(41.8)$ & $32(58.2)$ & \\
\hline$>1$ & $137(62.1)$ & $260(39.7)$ & \\
\hline $\begin{array}{l}\text { Extrathyroidal } \\
\text { extension }\end{array}$ & & & $0.0001^{\mathrm{b}}$ \\
\hline Positive & $25(18.9)$ & $107(81.1)$ & \\
\hline Negative & $130(42.3)$ & 177 (57.7) & \\
\hline $\begin{array}{l}\text { Lymph node } \\
\text { metastasis }\end{array}$ & & & $0.0057^{\mathrm{a}}$ \\
\hline Positive & $89(42.2)$ & $122(57.8)$ & \\
\hline Negative & $71(29.3)$ & $171(70.7)$ & \\
\hline TNM stage & & & $0.0001^{\mathrm{b}}$ \\
\hline $\mathrm{I}+\mathrm{II}$ & $135(6.9)$ & $183(12.7)$ & \\
\hline III+IV & $25(93.1)$ & $101(87.3)$ & \\
\hline
\end{tabular}

${ }^{\mathrm{a}} \mathrm{P}<0.01,{ }^{\mathrm{b}} \mathrm{P}<0.001$, Chi-square test. $\mathrm{PTC}$, papillary thyroid carcinoma.

added for $60 \mathrm{~min}$ at room temperature, followed by DAB development (DAB Substrate Chromogen system; Dako, Agilent Technologies, Shanghai, China) following standard staining protocol. The slides were fixed and images obtained with the Olympus IX71 inverted microscope using the DP2-BSW Olympus image acquisition software system. The results were confirmed by two experienced pathologists who were blinded to the clinicopathological data of the patients. The staining results were scored based on the percentage of stained tumor cell nuclei ( 0 , no staining; $1, \leq 10 \% ; 2,>10-50 \%$; and $3,>50 \%$ ).

Western blotting. The cells were lysed in RIPA buffer and boiled at $100^{\circ} \mathrm{C}$ for $10 \mathrm{~min}$ to obtain the protein lysates. Approximately $20 \mu \mathrm{g}$ protein lysates was extracted from each sample and separated by $10 \%$ SDS-PAGE. After being blocked in 5\% non-fat milk at room temperature for $1 \mathrm{~h}$, the protein concerned was probed using the primary antibodies: KMT5A (dilution, 1:1,000), p53 (dilution, 1:1,000; Santa Cruz Biotechnology, Shanghai, China), $\beta$-actin (dilution, 1:1,000; Cell Signaling Technology) at $4^{\circ} \mathrm{C}$ overnight, and then incubated with goat anti-rabbit $\mathrm{IgG}$ or goat anti-mouse $\operatorname{IgG}$ (dilution, 1:5,000; Cell Signaling Technology) at room temperature for $1 \mathrm{~h}$ and detected with enhanced chemiluminescence reagents (Thermo Fisher Scientific, Shanghai, China). The bands were visualized using 1-step ${ }^{\mathrm{TM}}$ NBT/BCIP reagents (Thermo Fisher Scientific, Rockford, IL, USA) and detected by the AlphaImager (Alpha Innotech, San Leandro, CA, USA). 
Table II. qRT-PCR primers of the target genes.

\begin{tabular}{lll} 
Target genes & \multicolumn{1}{c}{ Forward sequence $\left(5^{\prime}-3^{\prime}\right)$} & Reverse sequence $\left(5^{\prime}-3^{\prime}\right)$ \\
\hline KMT5A & CGCAAACTTACGGATTTCT & CGATGAGGTCAATCTTCATT \\
SREBP & GCGGAGCCATGGATTGCAC & CTCTTCCTTGATACCAGGCCC \\
SCD & TTCCTACCTGCAAGTTCTACACC & CCGAGCTTTGTAAGAGCGGT \\
FASN & AGCTGCCAGAGTCGGAGAAC & TGTAGCCCACGAGTGTCTCG \\
ACC & TAGATCCGTCAGAAAATGGGC & TACGGATATATTCTGCGTTGGC \\
$\beta$-actin & TGACGTGGACATCCGCAAAG & CTGGAAGGTGGACAGCGAGG
\end{tabular}

SREBP, sterol-regulatory element-binding factor; SCD, stearoyl-CoA desaturase; FASN, fatty acid synthase; ACC, acetyl-CoA carboxylase.

Cellular viability assays. Cell viability was determined using Cell Counting Kit-8 (CCK-8) assay according to the manufacturer's protocol. Briefly, the cells were seeded into 96 -well plates at $4 \times 10^{3}$ cells/well. An aliquot of $10 \mu \mathrm{l}$ CCK-8 solution was added to each well and the plate was incubated for $3 \mathrm{~h}$ at $37^{\circ} \mathrm{C}$. At the indicated time-points, the absorbance at $450 \mathrm{~nm}$ was assessed using a spectrophotometer. For each group, data from five wells were pooled. Each experiment was performed in triplicate.

Apoptosis assay. The apoptotic cells were assayed by flow cytometric analysis using the Annexin V-FITC/propidium iodide (PI) staining kit (BD Biosciences, Shanghai, China). The suspended cells were stained with $5 \mu \mathrm{l}$ Annexin V-FITC and $10 \mu \mathrm{l} \mathrm{PI}$ at room temperature for $15 \mathrm{~min}$. Flow cytometry was then used to immediately analyze the cells. The apoptotic cells were marked based on the Annexin $\mathrm{V}$ expression (Annexin $\mathrm{V}^{+} / \mathrm{PI}^{-}$and Annexin $\mathrm{V}^{+} / \mathrm{PI}^{+}$), quantified and compared to the controls from each group. The experiment was performed in triplicate.

Migration and invasion assays. The migration of cancer cells was assayed using $6.5-\mathrm{mm}$ diameter chambers with $8-\mu \mathrm{m}$ pore filters (Transwell, 24-well cell culture; BD Biosciences), while the invasion of cancer cells was assayed using chambers precoated with $20 \mu \mathrm{g}$ Matrigel (BD Biosciences). Both cell lines were suspended at $3 \times 10^{4}$ cells $/ \mathrm{ml}$ in serum-free media and then $0.1 \mathrm{ml}$ cell suspension was added to the upper chamber. Subsequently $0.6 \mathrm{ml}$ complete medium was added to the lower chamber. The chambers were incubated for $24 \mathrm{~h}$ at $37^{\circ} \mathrm{C}$ with $5 \% \mathrm{CO}_{2}$. After incubation, the filters were fixed and stained. The upper surface of the filters was scraped with cotton swabs to remove non-migrating cells. The experiments were repeated in triplicate wells and the number of migrating cells in five high-power fields per filter was counted microscopically at x200 magnification.

Cell cycle. In order to synchronize the cell cycle, the cells were harvested by trypsinization, washed in ice-cold phosphate-buffered saline (PBS) and fixed in 70\% ice-cold ethanol in PBS. After being resuspended in cold PBS, RNAase $(2 \mu \mathrm{g} / \mathrm{ml}$; BD Biosciences) was added and the cells were incubated at $37^{\circ} \mathrm{C}$ for $30 \mathrm{~min}$, followed by incubation in $400 \mu \mathrm{l} \mathrm{PI}(10 \mu \mathrm{g} / \mathrm{ml}$; BD Biosciences) for $40 \mathrm{~min}$ at room temperature. The DNA content was analyzed by flow cytometry and the cell population in the
G0, G1, S, G2 and M phase was analyzed. All experiments were performed in triplicate.

Lipid peroxidation (MDA) assay. Malondialdehyde (MDA) in the cells was determined using a lipid peroxidation MDA kit purchased from Beyotime Institute of Biotechnology (Shanghai, China) according to the manufacturer's instructions. Lysates from $2 \times 10^{6}$ cultured cells were collected with a mixture of ProteoJET Mammalian Cell Lysis Reagent (Fermentas, Shanghai, China). Afterwards the cells were homogenized in lysis solution on ice and then centrifuged at 12,000 $\mathrm{x} g$ for $10 \mathrm{~min}$. TBA reagent was added into each well containing $200 \mu \mathrm{l}$ standard and $200 \mu \mathrm{l}$ sample and incubated at $95^{\circ} \mathrm{C}$ for $60 \mathrm{~min}$. Subsequently the mix was cooled at room temperature in an ice bath for $10 \mathrm{~min}$. The activity of SOD was determined using a spectrophotometer at $\mathrm{Ex} / \mathrm{Em}=532 / 553 \mathrm{~nm}$ for fluorometric assay.

Flow cytometry. The reactive oxygen species (ROS) level in the K1 and TPC-1 cells after the knockdown of KMT5A was assessed by flow cytometry using Reactive Oxygen Species Assay kit (Beyotime Institute of Biotechnology) following the manufacturer's instructions. After being stained as described in the kit, ROS generation of cells was determined by flow cytometry (FC500; Beckman Coulter, Inc., Brea, CA, USA).

Statistical analysis. Statistical analyses were performed using GraphPad Prism 5.1 (GraphPad Software, Inc., La Jolla, CA, USA). Student's t-test and one-way ANOVA were performed to analyze the data. $\mathrm{P}<0.05$ was considered to indicate a statistically significant difference. The data are expressed as mean \pm SEM. All the experiments were performed at least three times.

\section{Results}

KMT5A is upregulated in the PTC samples and cell lines. To evaluate KMT5A expression in PTC tissue, qRT-PCR and western blotting, as well as IHC were performed in PTC and matched normal thyroid tissue specimens. The results revealed that KMT5A was transcriptionally upregulated in PTC samples $(\mathrm{P}<0.05$; Fig. 1A). Western blotting (Fig. 1B) and IHC ( $\mathrm{P}<0.001$; Fig. $1 \mathrm{C}$ and $\mathrm{D})$ analysis confirmed elevated KMT5A protein expression in PTC and low KMT5A protein expression in normal thyroid tissue specimens. To investigate whether KMT5A expression in PTC cell lines recapitulated its 
A

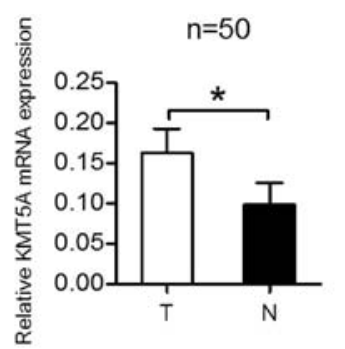

B

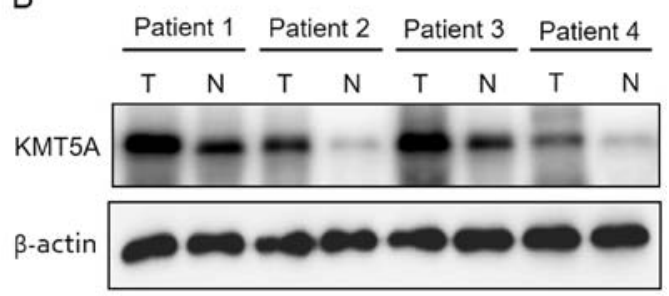

$\mathrm{C}$
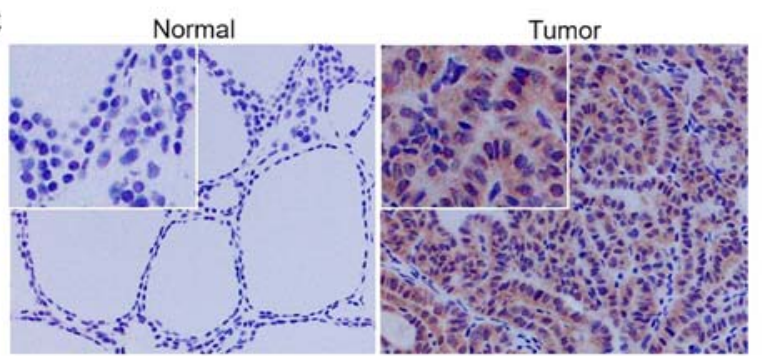

D

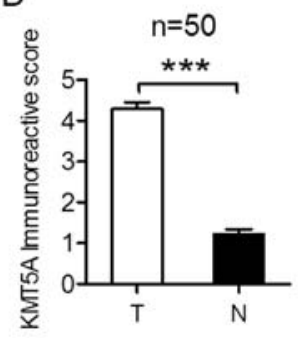

E
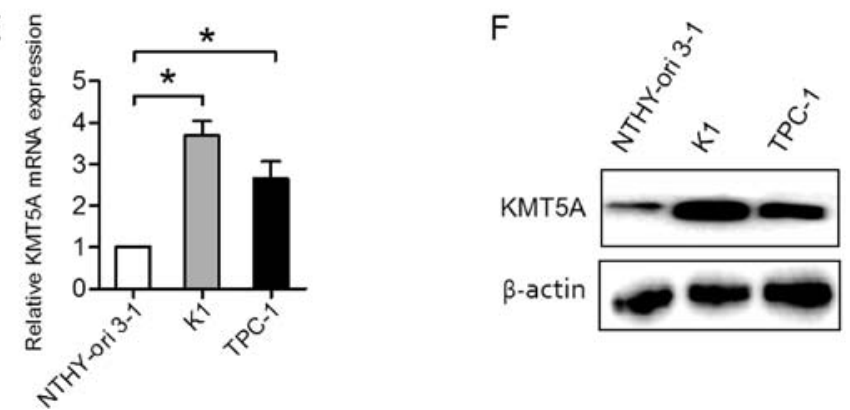

Figure 1. Expression of KMT5A in PTC tissue samples and PTC cell lines K1 and TPC-1. (A and B) Both mRNA and protein expression levels of KMT5A were elevated in PTC tissue samples ("P<0.05). (C and D) KMT5A was upregulated in PTC tissues relative to matched normal thyroid tissues by IHC ("P $<0.001)$ (original magnification, x200). (E and F) KMT5A in PTC cell lines K1 and TPC-1 were higher in both mRNA and protein levels by qRT-PCR and western blotting, compared with the normal thyroid epithelial Nthy-ori 3-1 cell line ( $\left.{ }^{*} \mathrm{P}<0.05\right)$. The results were normalized to $\beta$-actin expression. All the experiments were performed in triplicate. PTC, papillary thyroid carcinoma; IHC, immunohistochemical staining; T, PTC tissue samples; N, normal thyroid tissue specimens.

expression patterns observed in PTC patient tissues, human normal thyroid epithelial NTHY-ori 3-1 cells and PTC K1 and TPC-1 cells were tested. KMT5A was found to be overexpressed in $\mathrm{K} 1 \quad(\mathrm{P}<0.05)$ and $\mathrm{TPC}-1$ cells $(\mathrm{P}<0.05)$ compared to NTHY-ori 3-1 cells, both transcriptionally (Fig. 1E) and at protein level (Fig. 1F), which was consistent with the results in the PTC tissue samples.

Knockdown of KMT5A inhibits proliferation and induces apoptosis in PTC cells. To illuminate the efficacy of KMT5A on tumor cell proliferation and apoptosis, the expression of KMT5A was knocked down using targeted lentiviruses in $\mathrm{K} 1$ and TPC-1 cell lines. Transcriptional and protein knockdown was achieved using two separate lentiviral construct shRNA1 and shRNA2 as compared with shRNAcontrol-infected cells in both K1 (shRNA1, P<0.05; shRNA2, $\mathrm{P}<0.05)$ and TPC-1 (shRNA1, $\mathrm{P}<0.05$; shRNA2, $\mathrm{P}<0.01$ ) cells (Fig. 2A and B). Targeted knockdown of KMT5A resulted in attenuated proliferation in both $\mathrm{K} 1$ (shRNA1, $\mathrm{P}<0.001$; shRNA2, $\mathrm{P}<0.001$; Fig. 2C) and TPC-1 (shRNA1, $\mathrm{P}<0.001$; shRNA2, $\mathrm{P}<0.001$; Fig. 2D) cells after 48 h. Moreover, a significant increase in cell apoptosis measured by flow cytometry of PI/Annexin V-stained cells was observed in both K1 (shRNA1, P<0.001; shRNA2, P<0.001; Fig. 2E) and TPC-1 (shRNA1, $\mathrm{P}<0.05$; shRNA2, $\mathrm{P}<0.01$; Fig. $2 \mathrm{~F}$ ) cells using both shRNA1 and shRNA2 constructs.
Downregulated KMT5A suppresses migration and invasion in PTC cells. Next we evaluated the protumorigenic role of KMT5A in the migration and invasion of PTC cells. The results revealed that both TPC-1 (shRNA1, $\mathrm{P}<0.01$; shRNA2, $\mathrm{P}<0.05$ ) and $\mathrm{K} 1$ (shRNA1, $\mathrm{P}<0.01$; shRNA2, $\mathrm{P}<0.01$ ) cells transfected with KMT5A shRNA1 or shRNA2 displayed significantly decreased cell migration capacity than that of the shRNAcontrol vector (Fig. 3A and B). As shown in Fig. 3C and D, the invasion capacity was examined by Transwell assay and the invasive rate was markedly decreased in the KMT5A shRNA1- or shRNA2-transfected TPC-1 (shRNA1, P<0.05; shRNA2, $\mathrm{P}<0.01$ ) and $\mathrm{K} 1$ cells (shRNA1, $\mathrm{P}<0.05$; shRNA2, $\mathrm{P}<0.05)$ than in the shRNA-control transfected cells.

Loss of KMT5A arrests the G1/S phase in PTC cells. Several lines of evidence have revealed that KMT5A exerts crucial biological functions in modulating cell cycle and DNA damage response (17). We further verified the effect of KMT5A on the cell cycle of PTC cells by flow cytometry assay. As shown in Fig. 4, both K1 (Fig. 4A and B) (shRNA1, P<0.05; shRNA2, $\mathrm{P}<0.05$ ) and TPC-1 (Fig. 4C and D) (shRNA1, $\mathrm{P}<0.05$; shRNA2, P<0.05) cells transfected with KMT5A shRNA1 or shRNA2 had a significantly lower percentage of cells in the G1/S phase compared with the control group. The data revealed that the downregulation of KMT5A resulted in the cell cycle arrest of the PTC cells in the G1/S phase. 
A

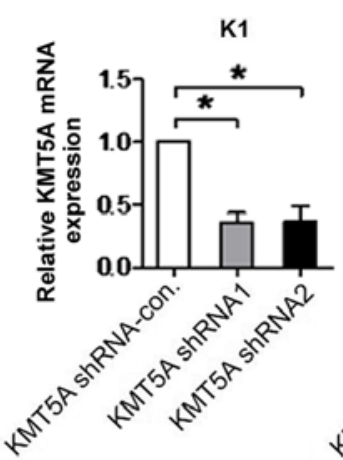

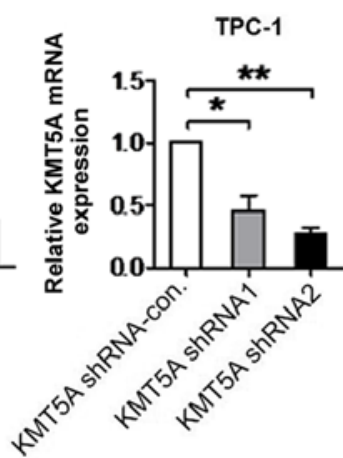
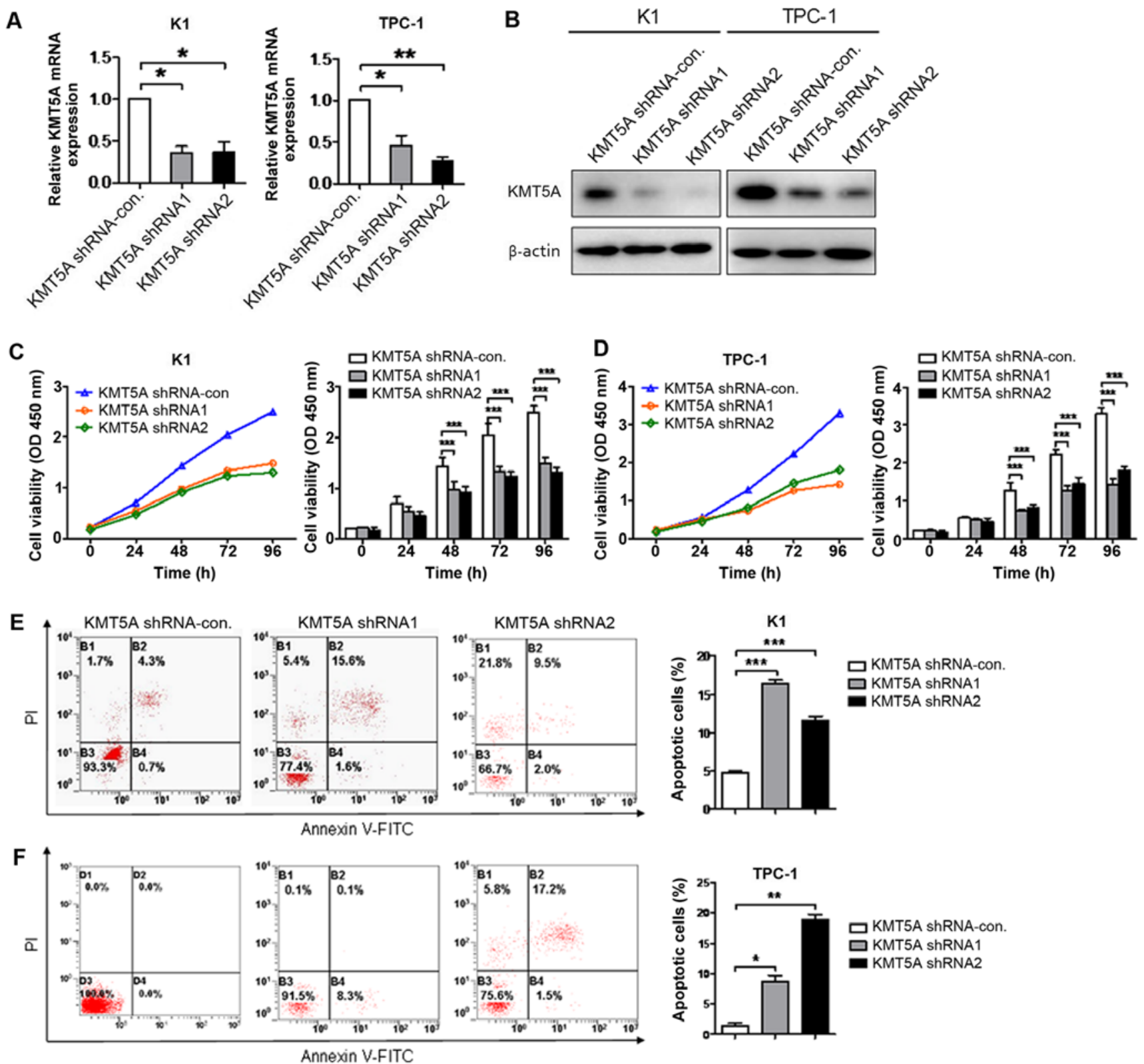

Figure 2. Biological function of KMT5A in the proliferation and apoptosis of K1 and TPC-1 cells in vitro. (A and B) K1 and TPC-1 cells were transfected with shRNA1 and shRNA2 to construct KMT5A-knockdown cell lines and KMT5A expression in the infected cells (K1, TPC-1-KMT5A shRNA1 and shRNA2) were confirmed by qPCR and western blotting (KMT5A shRNA-con, negative control). The results were normalized to $\beta$-actin mRNA expression. (C and D) CCK-8 cell proliferation assays were performed to determine KMT5A-knockdown K1 and TPC-1 cell proliferation. The results are presented as the mean \pm SEM from three independent experiments. (E and F) The apoptosis of KMT5A-knockdown K1 and TPC-1 cells was assessed by flow cytometry. The results are presented for infected cells relative to non-infected cells $\left({ }^{*} \mathrm{P}<0.05,{ }^{* *} \mathrm{P}<0.01,{ }^{* * * *} \mathrm{P}<0.001\right)$.

Targeting KMT5A reduces lipid metabolism of PTC cells. As a lysine methyltransferase enzyme, KMT5A regulates posttranslational modification of p53 at lysine 382 and cell cycle arrest functions of p53 (18). Our results revealed that KMT5A silencing led to the downregulation of p53 in the K1 and TPC-1 cells (Fig. 5A), which is consistant with previous studies. Recently, p53 has been reported to regulate metabolic pathways of breast cancer via sterol-regulatory elementbinding factor (SREBP) transcription factors, a key modulator gene of the lipid/cholesterol metabolism (19-22). To investigate the impact of KMT5A silencing on the lipid metabolism, we examined the expression of SREBP1 in KMT5A knockdown K1 and TPC-1 cells. As shown in Fig. 5B and C, the inhibition of KMT5A reduced SREBP1 in K1 and TPC-1 when compared with control transfected cells. Several lipid metabolism-related genes are controlled by SREBP1, such as stearoyl-CoA desaturase (SCD), fatty acid synthase (FASN) and acetyl-CoA carboxylase (ACC) (23). Subsequently, we revealed that KMT5A inhibition also decreased SCD, FASN and ACC transcriptional expression in the PTC cell lines (Fig. 5B and C).

Lipid peroxidation is the degradation of lipids that occurs as a result of oxidative damage. Polyunsaturated lipids are susceptible to oxidative attack, typically by ROS, resulting in a well-defined chain reaction with the production of end products such as MDA and ROS level. To further identify the effect of KMT5A on lipid metabolism, we assessed the lipid peroxidation level using MDA assay kit and the 


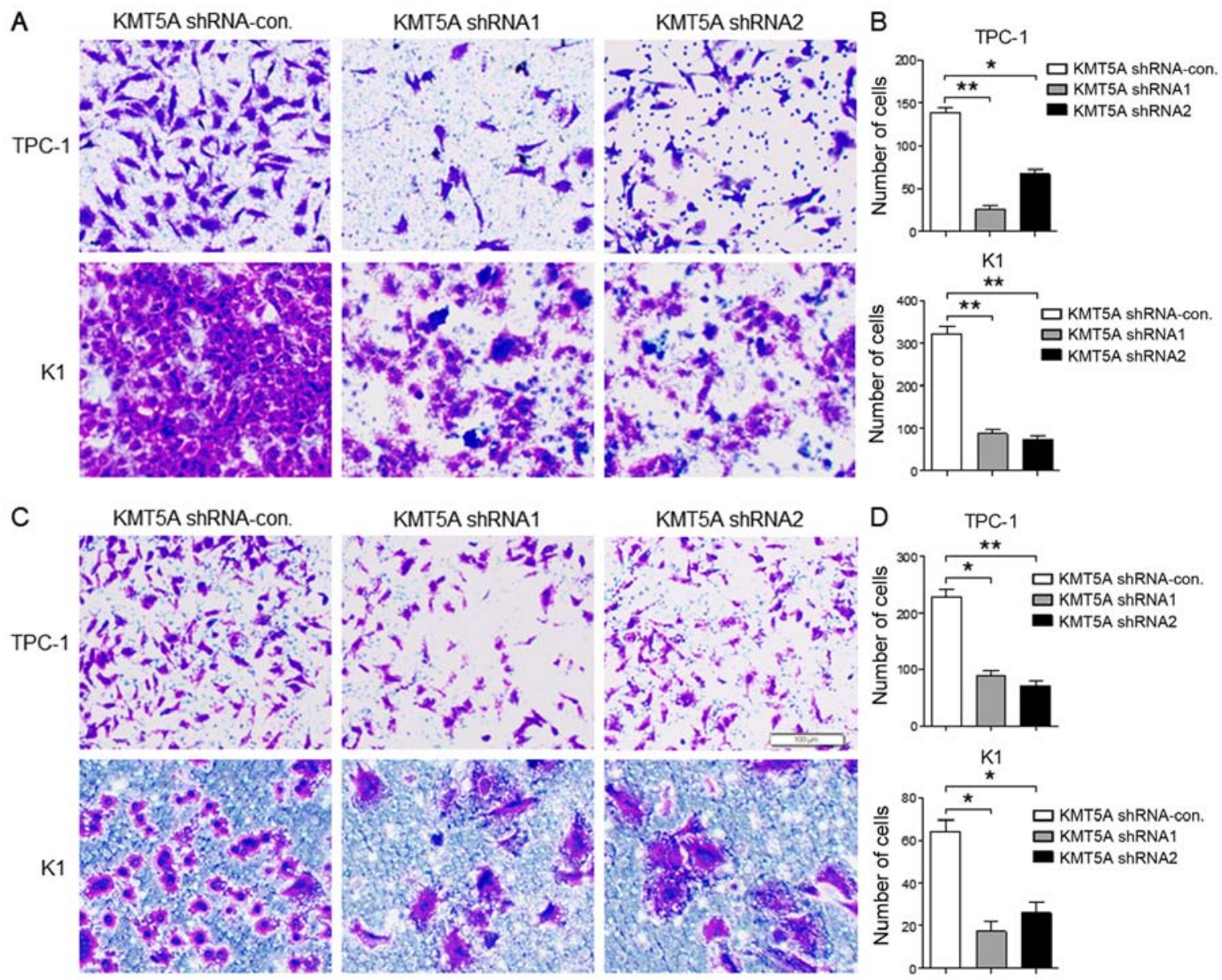

Figure 3. Inhibition of KMT5A suppresses migration and invasion in K1 and TPC-1 cell lines in vitro. Transwell assays were performed to determine migration (A and B) and invasion (C and D) of KMT5A-knockdown K1 and TPC-1 cells. The results are presented as the mean \pm SEM from three independent experiments $\left({ }^{*} \mathrm{P}<0.05,{ }^{* * *} \mathrm{P}<0.01\right)$.
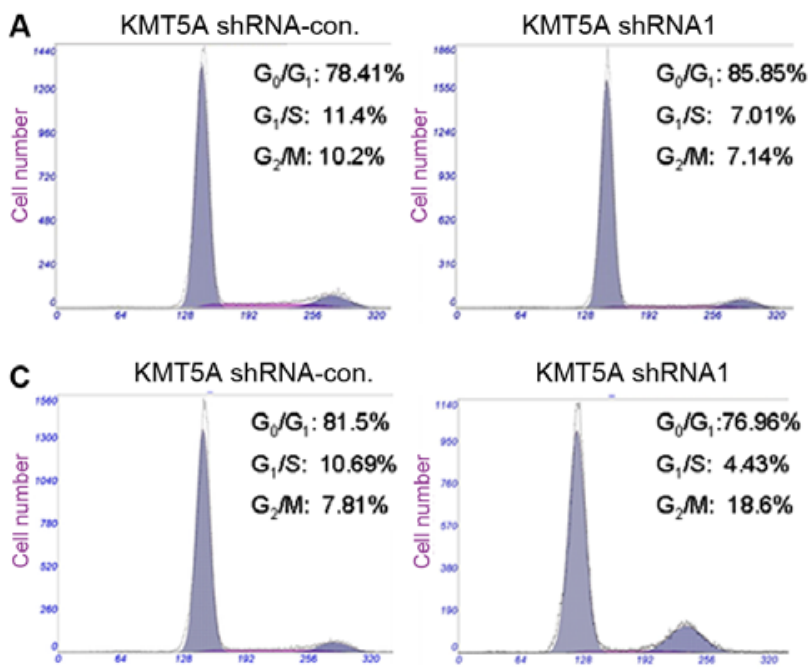
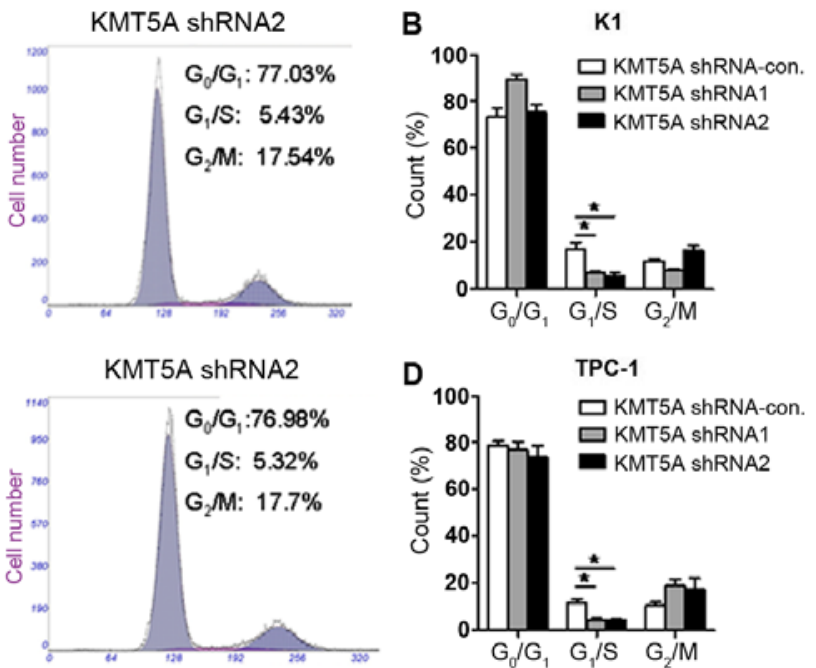

Figure 4. KMT5A arrests the G1 phase of the cell cycle of K1 and TPC-1 cells in vitro. The cell cycle distribution was analyzed to determine KMT5A-knockdown $\mathrm{K} 1$ (A and B) and TPC-1 (C and D) cells by flow cytometry. The results are presented as the mean \pm SEM from three independent experiments ("P<0.05).

ROS generation level using Reactive Oxygen Species assay kit. We demonstrated that silencing of KMT5A markedly decreased both the level of MDA and ROS in the K1 and TPC-1 cells compared with the shRNA-control-transfected cells (Fig. 5D-F).
Elevated KMT5A correlates with poor prognosis of PTC patients. To investigate the relationship between KMT5A and prognosis of PTC patients, we analyzed the KMT5A expression and clinical data in the TCGA database. Our results revealed that elevated KMT5A was significantly correlated 
A

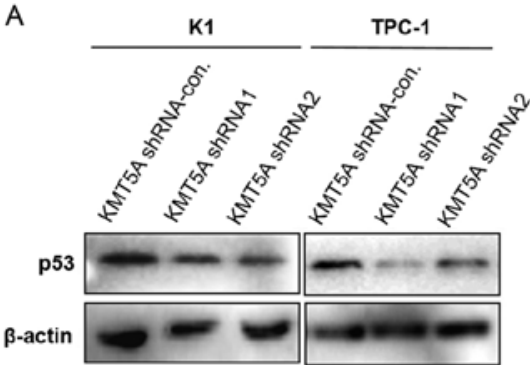

D

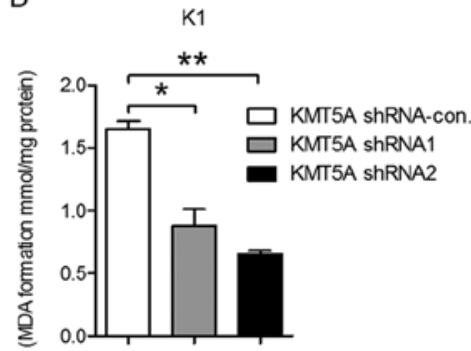

B

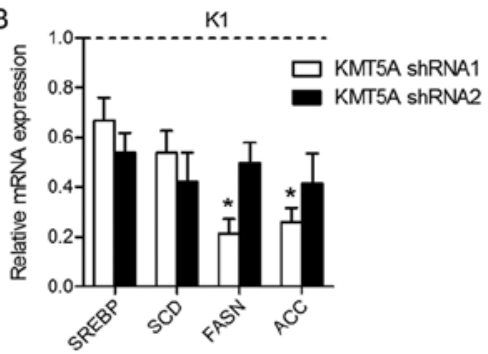

$\mathrm{E}$

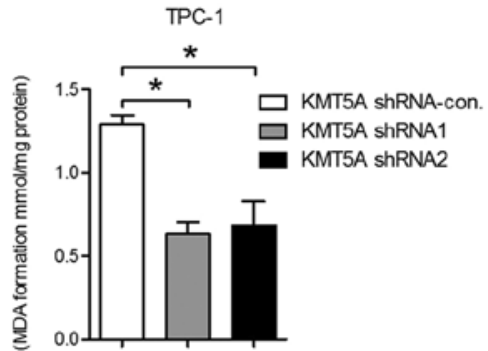

$\mathrm{C}$

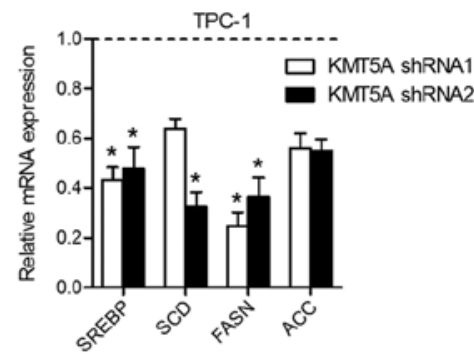

F

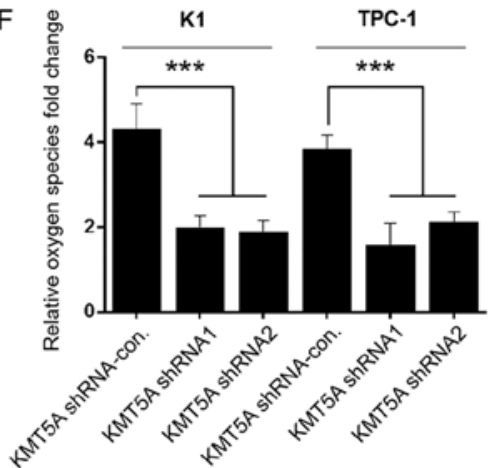

Figure 5. Knockdown of KMT5A inhibits p53 expression and lipid metabolism of K1 and TPC-1 cells in vitro. (A) The expression of p53 in KMT5A-knockdown K1 and TPC-1 cells was assessed by western blotting (KMT5A shRNA-con, negative control). (B and C) Relative levels of SREBP, stearoyl-CoA desaturase (SCD), fatty acid synthase (FASN) and acetyl-CoA carboxylase (ACC) in KMT5A-knockdown K1 and TPC-1 cells were examined by qPCR and normalized to $\beta$-actin mRNA expression $(" \mathrm{P}<0.05)$. The presented data were normalized to the expression of untreated control cell lines. (D and $\mathrm{E})$ Lipid peroxidation was measured in KMT5A-knockdown K1 and TPC-1 cells compared with shRNA-con cells. (F) ROS level was measured by flow cytometry in K1 and TPC-1 control cells (KMT5A shRNA-con.) and KMT5A-knockdown cells. The results are presented as the mean \pm SEM from three independent experiments $\left({ }^{*} \mathrm{P}<0.05,{ }^{* * *} \mathrm{P}<0.01,{ }^{* * * *} \mathrm{P}<0.001\right)$.

with extrathyroidal extension $(\mathrm{P}=0.0001)$, lymph node metastasis $(\mathrm{P}=0.0057)$ and advanced pathological stage $(\mathrm{P}=0.0001)$ in PTC tissues. However, KMT5A expression had no correlation with other clinicopathological paremeters, such as age $(\mathrm{P}=0.769)$, sex $(\mathrm{P}=0.259)$ or tumor size $(\mathrm{P}=0.29625)$ (Table $\mathrm{I})$. These data reveal that high KMT5A expression is associated with poor outcome in PTC patients.

\section{Discussion}

KMT5A, also known as SET8, is a monomethyltransferase required in cell cycle, replication, transcription and chromosome segregation (24). Previous studies have revealed that it plays an extensive role in multiple solid tumors. For example, it is related with the prognosis of breast cancer (25), it induces epithelial-mesenchymal transition and promotes metastasis of prostate cancer cells (26) and is associated with overall survival of gastric cancer patients (27). However, whether KMT5A has a function on PTC is still unknown.

In the present study we demonstrated that KMT5A is overexpressed in PTC tissue and cell lines K1 and TPC-1. To investigate the biological role in PTC, we used a lentivirus system to downregulate KMT5A expression in PTC cells. We found that knockdown of KMT5A inhibited the proliferation, migration and invasion of $\mathrm{K} 1$ and TPC-1 cells. In addition the inhibition of KMT5A induced apoptosis and arrested the G1 phase of the cell cycle in PTC cells. It is reported that KMT5A is almost undetectable in the $\mathrm{S}$ phase of the cell cycle and its impact on cell cycle progression is mainly through H4K20me1. Thus, the disruption of endogenous KMT5A may be accompanied by the suppression of H4K20 monomethylation and lead to cell cycle defects, chromatin decondensation and enlarged nuclei, indicating the essential role of KMT5A in DNA replication. Our results revealed that KMT5A may take part in the oncogenesis of PTC as a tumor gene. The relationship between KMT5A gene expression and extrathyroidal extension, lymph node metastasis, advanced pathological stage of papillary thyroid cancer patients from TCGA was in line with our in vitro data, indicating that KMT5A can be a novel marker for the prognosis of PTC patients.

Another finding in the present study was that the knockdown of KMT5A resulted in the decrease of p53, a crucial tumor suppressor maintaining metabolic homeostasis and orchestrating cellular stress responses. Recent research has reported that p53 and Hippo pathway cooperate on multiple levels to fine-tune SREBP activity and regulate cholesterol/lipid levels (28). The interaction between KMT5A and p53,p53 and SREBP raises the question of whether KMT5A regulates SREBP-mediated lipid metabolism. As expected, our results indicated that inhibition of KMT5A reduced the level of SREBP1 and its target genes (SCD, FASN and ACC), as well as suppressed the level of MDA and ROS generation in PTC cells, suggesting that KMT5A may regulate the lipid metabolism of PTC. However, whether the effect of KMT5A on the lipid metabolism is implemented via p53 needs more evidence to be elucidated.

In conclusion, a key issue we addressed for the first time is that histone methyltransferase KMT5A regulates the lipid metabolism of PTC. However, the underlying mechanism needs to be further explored. Additional investigation into other constituents of lipid metabolism may provide fresh 
insight into papillary thyroid carcinogenesis as well as reveal other potential therapeutic targets.

\section{Acknowledgements}

We would like to thank the University of Colorado Cancer Center Cell Bank for providing the PTC cell lines K1 and TPC-1. This study was partly supported by funds from the project sponsored by the Scientific Research Foundation for the Returned Overseas Chinese Scholars, State Education Ministry (to T.L.), the National Natural Science Foundation of China (nos. 81272934, 81572622 and 81772854 to Q.-H.J. and no. 81702753 to T.L.) and the Shanghai Science and Technology Commission Western Guide project (no. 14411962402 to D.-S.L.).

\section{References}

1. Morris LG, Tuttle RM and Davies L: Changing trends in the incidence of thyroid cancer in the United States. JAMA Otolaryngol Head Neck Surg 142: 709-711, 2016.

2. Xing M, Alzahrani AS, Carson KA, Shong YK, Kim TY, Viola D, Elisei R, Bendlová B, Yip L, Mian C, et al: Association between BRAF V600E mutation and recurrence of papillary thyroid cancer. J Clin Oncol 33: 42-50, 2015.

3. Carracedo A, Cantley LC and Pandolfi PP: Cancer metabolism: Fatty acid oxidation in the limelight. Nat Rev Cancer 13 227-232, 2013.

4. Santos CR and Schulze A: Lipid metabolism in cancer. FEBS J 279: 2610-2623, 2012

5. Baenke F, Peck B, Miess H and Schulze A: Hooked on fat: The role of lipid synthesis in cancer metabolism and tumour development. Dis Model Mech 6: 1353-1363, 2013.

6. von Roemeling CA, Marlow LA, Pinkerton AB, Crist A, Miller J, Tun HW, Smallridge RC and Copland JA: Aberrant lipid metabolism in anaplastic thyroid carcinoma reveals stearoyl CoA desaturase 1 as a novel therapeutic target. J Clin Endocrinol Metab 100: E697-E709, 2015.

7. Uddin S, Siraj AK, Al-Rasheed M, Ahmed M, Bu R, Myers JN, Al-Nuaim A, Al-Sobhi S, Al-Dayel F, Bavi P, et al: Fatty acid synthase and AKT pathway signaling in a subset of papillary thyroid cancers. J Clin Endocrinol Metab 93: 4088-4097, 2008.

8. Li Z, Nie F, Wang S and Li L: Histone H4 Lys 20 monomethylation by histone methylase SET8 mediates Wnt target gene activation. Proc Natl Acad Sci USA 108: 3116-3123, 2011.

9. Abbas T, Shibata E, Park J, Jha S, Karnani N and Dutta A: $\mathrm{CRL} 4{ }^{\mathrm{Cd} 2}$ regulates cell proliferation and histone gene expression by targeting PR-Set7/Set8 for degradation. Mol Cell 40: 9-21, 2010.

10. Centore RC,Havens CG, Manning AL, Li JM,Flynn RL, Tse A, Jin J, Dyson NJ, Walter JC and Zou L: CRL4 ${ }^{\mathrm{Cdt} 2}$-mediated destruction of the histone methyltransferase Set 8 prevents premature chromatin compaction in S phase. Mol Cell 40: 22-33, 2010.

11. Houston SI, McManus KJ, Adams MM, Sims JK, Carpenter PB, Hendzel MJ and Rice JC: Catalytic function of the PR-Set7 histone $\mathrm{H} 4$ lysine 20 monomethyltransferase is essential for mitotic entry and genomic stability. J Biol Chem 283: 19478-19488, 2008.
12. Oda H, Hübner MR, Beck DB, Vermeulen M, Hurwitz J, Spector DL and Reinberg D: Regulation of the histone H4 monomethylase PR-Set7 by CRL4 ${ }^{\text {Cdt2 }}$-mediated PCNA-dependent degradation during DNA damage. Mol Cell 40: 364-376, 2010.

13. Wu S, Wang W, Kong X, Congdon LM, Yokomori K, Kirschner MW and Rice JC: Dynamic regulation of the PR-Set7 histone methyltransferase is required for normal cell cycle progression. Genes Dev 24: 2531-2542, 2010.

14. Shi X, Kachirskaia I, Yamaguchi H, West LE, Wen H, Wang EW, Dutta S, Appella E and Gozani O: Modulation of p53 function by SET8-mediated methylation at lysine 382. Mol Cell 27: 636-646, 2007

15. Yang F, Sun L, Li Q, Han X, Lei L, Zhang H and Shang Y: SET8 promotes epithelial-mesenchymal transition and confers TWIST dual transcriptional activities. EMBO J 31: 110-123, 2012.

16. Liao T, Wen D, Ma B, Hu JQ, Qu N, Shi RL, Liu L, Guan Q, Li DS and Ji QH: Yes-associated protein 1 promotes papillary thyroid cancer cell proliferation by activating the ERK/MAPK signaling pathway. Oncotarget 8: 11719-11728, 2017.

17. Wang Z, Dai X, Zhong J, Inuzuka H, Wan L, Li X, Wang L, Ye X, Sun L, Gao D, et al: SCF ${ }^{\beta-T R C P}$ promotes cell growth by targeting PR-Set7/Set8 for degradation. Nat Commun 6: 10185, 2015.

18. Swain JF, Dinler G, Sivendran R, Montgomery DL, Stotz M and Gierasch LM: Hsp70 chaperone ligands control domain association via an allosteric mechanism mediated by the interdomain linker. Mol Cell 26: 27-39, 2007.

19. Cook KL, Soto-Pantoja DR, Clarke PA, Cruz MI, Zwart A, Wärri A, Hilakivi-Clarke L, Roberts DD and Clarke R: Endoplasmic reticulum stress protein GRP78 modulates lipid metabolism to control drug sensitivity and antitumor immunity in breast cancer. Cancer Res 76: 5657-5670, 2016.

20. Sorrentino G, Ruggeri N, Specchia V, Cordenonsi M, Mano M, Dupont S, Manfrin A, Ingallina E, Sommaggio R, Piazza S, et al: Metabolic control of YAP and TAZ by the mevalonate pathway. Nat Cell Biol 16: 357-366, 2014.

21. Freed-Pastor WA, Mizuno H, Zhao X, Langerød A, Moon SH, Rodriguez-Barrueco R, Barsotti A, Chicas A, Li W, Polotskaia A, et al: Mutant p53 disrupts mammary tissue architecture via the mevalonate pathway. Cell 148: 244-258, 2012.

22. Li X, Wu JB, Chung LW and Huang WC: Anti-cancer efficacy of SREBP inhibitor, alone or in combination with docetaxel, in prostate cancer harboring p53 mutations. Oncotarget 6: 41018-41032, 2015.

23. Horton JD, Goldstein JL and Brown MS: SREBPs: Activators of the complete program of cholesterol and fatty acid synthesis in the liver. J Clin Invest 109: 1125-1131, 2002.

24. Dulev S, Tkach J, Lin S and Batada NN: SET8 methyltransferase activity during the DNA double-strand break response is required for recruitment of 53BP1. EMBO Rep 15: 1163-1174, 2014.

25. Liu B, Zhang X, Song F, Liu Q, Dai H, Zheng H, Cui P, Zhang L, Zhang $\mathrm{W}$ and Chen $\mathrm{K}$ : A functional single nucleotide polymorphism of SET8 is prognostic for breast cancer. Oncotarget 7: 34277-34287, 2016.

26. Hou L, Li Q, Yu Y, Li M and Zhang D: SET8 induces epithelial mesenchymal transition and enhances prostate cancer cell metastasis by cooperating with ZEB1. Mol Med Rep 13: 1681-1688, 2016.

27. Shi XL, Guo ZJ, Wang XL, Liu XL and Shi GF: SET8 expression is associated with overall survival in gastric cancer. Genet Mol Res 14: 15609-15615, 2015.

28. Aylon Y and Oren M: The Hippo pathway, p53 and cholesterol. Cell Cycle 15: 2248-2255, 2016. 